IUGR/stillbirth. Women with RFM and risk factors for IUGR/ stillbirth had statistically significant more pre-term deliveries and more babies born with low birth weight compared to women with no risk factors (Table 1).

Abstract PP.83 Table 1 Pregnancy outcomes compared between women with/without risk factors for IUGR/stillbirth.

\begin{tabular}{lll}
\hline Pregnancy outcomes (\%) & $\begin{array}{l}\text { With risk factors for } \\
\text { IUGR/stillbirth } \mathbf{n}=\mathbf{4 2}\end{array}$ & $\begin{array}{l}\text { With no risk factors for IUGR/ } \\
\text { stillbirth n= 76 }\end{array}$ \\
\hline Livebirth- term & $85.7^{*}$ & $98.7^{*}$ \\
Livebirth- preterm & $11.9^{*}$ & $0^{*}$ \\
Stillbirth & 1.24 & 1.3 \\
Birth weight $\geq 2500 \mathrm{~g}$ & $76.2^{*}$ & $98.7^{*}$ \\
Birth weight $1500-2499 \mathrm{~g}$ & $19^{*}$ & $1.3^{*}$ \\
Birth weight $\leq 1499 \mathrm{~g}$ & 2.4 & 0 \\
\hline
\end{tabular}

${ }^{*} p<0.05$

Although women with recurrence of RFM had more pre-term and stillbirth deliveries and babies with low birth weight compared to women presenting once with RFM, the results failed to show statistical significance. There was no difference in pregnancy outcomes between women with reassuring CTG and ongoing perception of RFM compared with those women with reassuring CTG and perceived fetal activity during the assessment (Table 1).

Conclusion Risk factors for IUGR/stillbirth are significant determinants of poor pregnancy outcomes in women presenting with RFM.

\section{PP.84 THE IMPACT OF MATERNAL AGE AND CHORIONICITY ON OBSTETRIC OUTCOME IN TWIN PREGNANCY}

doi:10.1136/archdischild-2013-303966.359

'AM O' Mahoney, ${ }^{2}$ S Meaney, ${ }^{3} \mathrm{~K}$ O' Donoghue. 'School of Medicine, University College Cork, Ireland; ${ }^{2}$ National Perinatal Epidemiology Centre, University College Cork, Ireland; ${ }^{3}$ Dept. of Obstetrics and Gynaecology, University College Cork, Ireland

Introduction The number of twins born in Ireland has increased from 11.8 sets per 1,000 live births in 1988 to 17.9 in 2011. This study aims to investigate the impact of advanced maternal age and chorionicity on obstetric outcome in twin pregnancies.

Methods A retrospective cohort study of all twin pregnancies delivered from 2009 to 2011 in a large, tertiary hospital ( 8,000 deliveries per annum) in the Republic of Ireland was conducted. Birth registers and clinic records were reviewed to examine obstetric outcomes.

Results Of the 523 twin pregnancies included in the study $9.6 \%$ $(n=50)$ of mothers were $\geq 40$ years and $47.5 \%(n=247)$ were nulliparous. Advanced maternal age, mother aged $\geq 40$ years, was associated with increased ART (52\% vs. $25.2 \% ; p<0.001)$ and increased caesarean delivery $(78.0 \%$ vs. $60.9 \%$; $<<0.001)$. Differences between the age groups were noted for GDM (9.8\% vs. $3.1 \%$; $=0.007)$ and PET/PIH (30.8\% vs. $13.8 \%$; $p<0.001) .16 .3 \%(n=87)$ of twins were monochorionic who had a higher incidence of PTD $(65.1 \% \mathrm{~V} 47.9 \%$, $\mathrm{p}=<0.001)$ and iatrogenic reasons for PTD $(69.4 \% \mathrm{~V} 52.4 \%$, $p=0.006)$ than dichorionic twins.

Conclusion As a greater number of women are delaying childbearing, and with advances in ART, there are considerable more first time births, including sets of twins, to older women. The findings of this study indicate that advanced maternal age and nulliparity were associated with adverse obstetric outcome in twin pregnancies.

\section{PP.85 EVALUATION OF THE EARLY PREGNANCY CLINIC - INCLUDING PATIENT AND STAFF PERSPECTIVES ON THE QUALITY OF SERVICE PROVISION}

doi:10.1136/archdischild-2013-303966.360

CM Coakley, K O'Donoghue. Department of Obstetrics and Gynaecology, Cork University Maternity Hospital, University College Cork, Cork, Ireland
Objectives Early pregnancy loss occurs in 15-20\% of clinicallyconfirmed pregnancies. A dedicated early pregnancy clinic (EPC) with provision of diagnostic services such as transvaginal ultrasonography and on-site laboratory investigations contributes to a reduction of repeated assessments for ectopic pregnancy and miscarriage, and is a necessary facility in every maternity unit. Our objectives were to (i) examine clinical records for EPC attendances, (ii) obtain staff and patient feedback to identify areas for improvement and (iii) review EPC incident reports and complaint forms.

Study design A retrospective audit was performed on EPC attendances during 2011 using clinic databases; subsequently categorised according to referral sources, indications, new cases, diagnosis and management. Incident and complaint forms were analysed for recurrent themes. Staff and patient satisfaction questionnaires were distributed obtaining a random sample of 70 patients and all 24 staff.

Results There were 5,233 attendances during 2011, with GPs the main source of referrals. Emergency room referrals contributed the highest workload for repeat scans and blood tests. While $95 \%$ of staff expressed job satisfaction, $83 \%$ reported encountering stress attributed to individual workload and co-worker conflict, while junior medical staff were concerned about lack of training. Overall, $95 \%$ of patients were satisfied with staff and clinic organisation, with many instead highlighting concerns with the physical space and ability to overhear confidential medical information. All 6 patient complaints related to communication issues.

Conclusion High levels of job satisfaction and good teamwork were identified and we received largely positive feedback. Areas of improvement include staff workload and training.

\section{PP.86 OUTCOME OF PREGNANCIES WITH UTERINE LEIOMYOMA: A POPULATION BASED RETROSPECTIVE STUDY}

doi:10.1136/archdischild-2013-303966.361

D Mitra, S Bhatti, J Iskaros. University College London Hospital (UCLH), London, UK

Uterine leiomyoma (fibroids), which are often found in childbearing age, can lead to complications in pregnancy and hence are considered to be of high risk.

Aims and Objectives To observe the effects of fibroids in pregnancy and look at associated maternal and fetal complications.

Methods A population-based retrospective study was conducted in a central London tertiary hospital. Women were identified from first Trimester Ultrasound scans (done between January and December 2011) database using search word 'Fibroids' and their pregnancy outcome noted from the local obstetric electronic system.

Results 201 women were identified, of which 42 did not deliver at our hospital, making final sample size 159 . We looked into the incidence of preterm birth (PTB), low birth weight (LBW) babies $(<2500 \mathrm{gm})$, operative delivery (abdominal and vaginal) and the occurrence of Postpartum Haemorrhage (PPH).

Among the 159 women, $13(8.17 \%)$ delivered preterm ( $<37$ weeks), while $4(2.53 \%)$ had miscarriages. The occurrence of LBW was $16(10.06 \%)$. Spontaneous vaginal delivery (SVD) occurred in $53(33.33 \%)$ cases, instrumental vaginal delivery (forceps and ventouse) in 32 (20.12\%) cases, and Caesarean section (CS) was performed in 70 cases $(44.02 \%)$. The primary indications for CS included failure to progress in labour 11 (15.71\%), fetal distress 15 (21.43\%), previous Caesarean section $15(21.43 \%)$, and malpresentation 12 (17.14\%). Minor and major PPH were observed in 41 $(25.78 \%)$ and $6(3.77 \%)$ cases, respectively.

Conclusion We found pregnancies with fibroids to be statistically correlated with increased Caesarean section and PPH. These results highlight the necessity for good antenatal, intrapartum, and postpartum care for optimum outcome. 


\section{PP.88 IDENTIFICATION OF CLINICALLY IMPORTANT COMPONENTS OF MATERNAL AND NEONATAL COMPOSITE OUTCOMES TO ASSESS THE EFFECT OF TIMING OF DELIVERY IN WOMEN WITH MILD TO MODERATE PRE-ECLAMPSIA AT 34 TO 37 WEEKS}

doi:10.1136/archdischild-2013-303966.362

1,2F Fong, 1,2E Rogozinska, ${ }^{1,2} \mathrm{~J}$ Allotey, ${ }^{3} \mathrm{~S}$ Kempley, ${ }^{4} \mathrm{D}$ Shah, 1,2.5 Thangaratinam. 'Women's Health Research, Unit, Barts and the London School of Medicine and Dentistry, Queen Mary University London, London, UK; ${ }^{2}$ Centre of Primary Care and Public Health, Barts and the London School of Medicine and Dentistry, OMUL, London, UK; ${ }^{3}$ Centre for Paediatrics, Blizard Institute, Barts and the London School of Medicine, OMUL, London, UK; ${ }^{4} B$ arts and the London Children's Hospital, QMUL, London, UK ${ }^{5}$ Multidisciplinary Evidence Synthesis Unit (MESH), OMUL, London, UK

Background Pre-eclampsia is associated with maternal and neonatal morbidity and mortality. Delivery is often expedited to minimise maternal complications at the risk of prematurity-related complications in the baby. The important outcomes to be evaluated in the management of pregnant women with pre-eclampsia between 34 to 37 weeks gestation is unclear.

Objective To identify the clinically important components of maternal and neonatal composite outcomes in the management of women with mild to moderate pre-eclampsia between 34 to 37 weeks by a Delphi survey of experts.

Methods A two-generational Delphi method was used to prioritise the maternal and neonatal outcomes that are considered to be important in the timing of delivery in women with mild to moderate pre-eclampsia.

Results The maternal outcomes questionnaire was sent to 20 participants and the neonatal outcomes questionnaire to 24 participants. $18 / 20$ participants $(90 \%)$ responded in the first round for maternal outcomes and $18 / 24$ participants (75\%) for neonatal outcomes. In the second round 16/17 eligible participants (94\%) responded for maternal outcomes and $18 / 18$ participants $(100 \%)$ for the neonatal outcomes. 21 maternal and 24 neonatal outcomes were evaluated in the first round and 17 maternal and 25 neonatal outcomes in the second round. We identified 17 and 17 components to be clinically important for development of the maternal and neonatal composite outcomes respectively. If accepted, we will be able to provide full details of the relevant outcomes.

Conclusion The composite maternal and neonatal outcomes will allow evaluation of the effect of intervention to reduce mortality and morbidity.

\section{PP.89 PRETERM PRE-LABOUR RUPTURE OF MEMBRANES (PPROM) - WHEN CLINICIANS ASPIRE TO FIND A BALANCE}

doi:10.1136/archdischild-2013-303966.363

\section{S Islam, S Hickey, S Hamilton. Calderdale Royal Hospital, HALIFAX, UK}

Introduction and objectives Preterm Pre-labour Rupture of Membranes complicates $2 \%$ of all pregnancies and is related to $40 \%$ of all preterm deliveries with significant neonatal and maternal risks. Balancing the risks between prematurity and maternal/fetal infection can pose difficult dilemmas for obstetricians. This study collected data on PPROM-delivery interval, maternal and fetal outcomes and examined the impact of inter-clinician variation in practise on clinical outcomes.

Methodology This retrospective observational study included 48 women with PPROM before 36 weeks of gestation between January 2011 and April 2012 at Calderdale Royal Hospital.
Results All patients with confirmed PPROM received erythromycin and steroid. Incidence of gestation $<34$ weeks at PPROM was $40 \%$; of delivery at $<34$ weeks was $28 \%$. Prolongation of pregnancy of $2-7$ days occurred in 52\%, 7-14 days in 2\% and $>14$ days in $13 \%$. Timing of induction of labour (IOL) varied from 34 to $36+6$ weeks although without any adverse outcomes. There were two neonatal deaths (NND) following spontaneous labour

Conclusion Prolongation of pregnancy of 48 hours or more occurred in a significant percentage of patients with conservative management. Prematurity played a crucial role in the two instances of NND. It is not clear that outcomes would have been different with different clinical management. This series shows that PPROM caries significant threat to perinatal outcome even with optimum obstetric care.

\section{PP.90 RISK FACTORS ASSOCIATED WITH INTRAUTERINE DEATHS - OUR EXPERIENCE AT DISTRICT GENERAL HOSPITAL}

doi:10.1136/archdischild-2013-303966.364

HZ Ling, 0 Mulki, R Hamid. Ealing Hospital NHS Trust, Southall, UK

Objective To identify the relationship between intrauterine death (IUD) and various demographics, medical and obstetrics risk factors relating to the local population in Ealing.

Methods There were 47 booked, singleton IUD cases amongst 7464 births in Ealing Hospital between April 2010 to September 2012. We retrospectively analysed the medical and obstetrics risk factors of IUDs (fetus $\geq 24 / 40$ with absent fetal heart activity inutero) in the local population.

Results IUD occurred in $0.63 \%$ of all birth. Females of Asian origin are at increased risk of IUD (45\%) compared to other ethnicities. Lifestyle risks like smoking and alcohol posed no significant increase in risk to the Ealing population, and consanguineous relationships only accounted for $8.5 \%$ of the cases. IUD was most prevalent amongst the young (20-25) primips (59.5\%) with BMI > $25(68 \%)$, mostly occurring between $37-40$ weeks (47.7\%). Majority of them were booked $\leq 12 / 40$ (63.8\%), had Dating and Anomaly scans, $(87.2 \%, 89.3 \%$ respectively), low risk on antenatal screening (59.5\%). Interestingly, previous caesarean section (6.3\%) was the most prevalent in obstetrics history. $68 \%$ had presented antenatally with reduced fetal movements, vaginal bleeding or abdominal pain. Maternal conditions like Diabetes (4.2\%), Obstetrics Cholestasis $(2.1 \%)$ and Preeclampsia (10\%) were not strongly associated with the IUD cases, indicating robust antenatal surveillance we provide to high risk women.

Conclusion While further studies aiming to modify IUD risk factors are needed, careful planning on timing of induction of labour should be carried out specifically in young, Asian primips with BMI $>25$ who present antenatally with significant episode(s).

\section{PP.91 RUPTURE UTERUS FOLLOWING PREVIOUS CAESAREAN SECTION IN THE SECOND AND EARLY THIRD TRIMESTER OF SUBSEQUENT PREGNANCY}

doi:10.1136/archdischild-2013-303966.365

S Hirsi-Farah, F Ajibade, P Street. Royal Berkshire Hospital, Reading, UK

Three cases of antenatal/pre-labour uterine rupture in women with scarred uterus.

Rupture uterus following lower segment caesarean section is rare occurrence.

Rupture uterus is linked with abnormal implantation of the placenta, and endometrial deficiency. In 10\% there's invasive placentation where placenta invades to deeper layers increasing the risks of bleeding; prematurity; SB/NND; hysterectomy and ICU admission. 